\title{
RELAÇÃO ENTRE EVENTOS TROMBÓTICOS E INFECÇÃO PELA SARS-COV-2: UMA REVISÃO DE LITERATURA
}

\section{RELATIONSHIP BETWEEN THROMBOTIC EVENTS AND SARS-COV-2 INFECTION: A LITERATURE REVIEW}

\author{
Tahissa Frota Cavalcante ${ }^{1} *$ Cristefânia Meirú de Lima ${ }^{2} *$ José Erivelton de Souza Maciel Ferreira $^{3} *$ \\ Osmar Rodrigues Paixão Neto ${ }^{4} *$ Rafaella Pessoa Moreira $^{5} *$ Viviane Nóbrega * Goularte Azevedo ${ }^{6}$
}

\begin{abstract}
RESUMO
Objetivo: apresentar o conhecimento produzido sobre eventos trombóticos e sua relação com a infecção pela SARSCoV-2. Método: estudo do tipo revisão integrativa da literatura, realizado nos meses de maio e junho de 2020 , em quatro bases de dados/bibliotecas virtuais/portais e buscadores acadêmicos, com as seguintes palavras-chave: coronavírus, SARS and thrombus (Pubmed, Cochrane and Science Direct) e Vírus da SARS and Transtornos da Coagulação Sanguínea (Biblioteca Virtual da Saúde). Após a busca, foram encontrados 263 estudos e com o emprego dos critérios de inclusão e exclusão restaram 59 trabalhos. Resultados: os trabalhos mostraram que as chances de ocorrência de eventos trombóticos aumentam em pessoas mais idosas e com comorbidades. Além disso, os eventos trombóticos mais prevalentes nos pacientes acometidos pela doença foram trombose pulmonar microvascular, tromboembolismo venoso de membros inferiores, síndrome coronariana aguda e acidente vascular cerebral. A avaliação e acompanhamento de pacientes, principalmente os internados em Unidade de Terapia Intensiva, no tocante ao seu estado inflamatório e os seus marcadores de hipercoagulabilidade são importantes para a detecção precoce e tomada de decisão para a administração de terapias anticoagulantes. A enfermagem é primordial para o acompanhamento e monitoramento desses pacientes. Considerações finais: embora a incidência dos eventos trombóticos em pessoas com COVID-19 em âmbito mundial já esteja melhor descrita na literatura, ainda é necessário que se produzam mais estudos clínicos e epidemiológicos para melhor explicar essa relação; é importante ainda para que se possa determinar se de fato essa doença pode ser classificada como pertencente ao grupo de doenças vasculares.
\end{abstract}

Palavras-chave: SARS-CoV-2; Infecções por Coronavirus; Trombo; Enfermagem; Enfermagem de Cuidados Críticos.

\begin{abstract}
Objective: to present the knowledge produced about thrombotic events and their relationship with SARS-CoV-2 infection. Method: an integrative literature review study, carried out in May and June 2020, in four databases/virtual libraries/portals and academic search engines, with the following keywords: coronavirus, SARS and thrombus (Pubmed, Cochrane and Science Direct) and SARS Virus and Blood Coagulation Disorders (Virtual Health Library). After the search, 263 studies were found and with the use of inclusion and exclusion criteria, 59 works remained. Results: the studies showed that the chances of occurrence of thrombotic events increase in older people with comorbidities. In addition, the most prevalent thrombotic events in patients affected by the disease were microvascular pulmonary thrombosis, lower limb venous thromboembolism, acute coronary syndrome and stroke. The assessment and follow-up of patients, especially those hospitalized in the Intensive Care Unit, regarding their inflammatory state and their hypercoagulability markers are important for early detection and decision-making for the administration of anticoagulant therapies. Nursing is essential for the follow-up and monitoring of these patients. Final considerations: although the incidence of thrombotic events in people with COVID-19 worldwide is already better described in the literature, it is still necessary to produce more clinical and epidemiological studies to better explain this relationship; it is also important to determine whether this disease can actually be classified as belonging to the group of vascular diseases.
\end{abstract}

Keywords: SARS-CoV-2; Coronavirus Infections; Thrombosis; Nursing; Critical Care Nursing.

\footnotetext{
${ }^{1}$ Enfermeira. Doutora em Enfermagem. Professora do curso de Enfermagem e do Programa de Pós-Graduação em Enfermagem da Universidade da Integração Internacional da Lusofonia Afro-Brasileira (UNILAB), Redenção-Ceará, Brasil. Orcid: https://orcid.org/0000-0002-7758-4273

2 Acadêmica do curso de graduação em Enfermagem da Universidade da Integração Internacional da Lusofonia Afro-Brasileira (UNILAB), Redenção-CE, Brasil. Orcid: https://orcid.org/0000-0001-8919-4988

${ }^{3}$ Enfermeiro. Especialista em Enfermagem Cirúrgica. Pós-graduando lato sensu em Enfermagem do Trabalho. Mestrando em Enfermagem da Universidade da Integração Internacional da Lusofonia Afro-Brasileira (UNILAB), Redenção-Ceará, Brasil. Orcid: https://orcid.org/0000-0003-2668$\underline{7587}$

${ }^{4}$ Acadêmico do curso de graduação em Enfermagem da Universidade da Integração Internacional da Lusofonia Afro-Brasileira (UNILAB), Redenção-Ceará, Brasil. Orcid: https://orcid.org/0000-0003-4183-6018

${ }^{5}$ Enfermeira. Doutora em Enfermagem. Professora do curso de Enfermagem e do Programa de Pós-Graduação em Enfermagem da Universidade da Integração Internacional da Lusofonia Afro-Brasileira (UNILAB), Redenção-Ceará, Brasil. Orcid: https://orcid.org/0000-0003-2341-7936

${ }^{6}$ Enfermeira. Especialista em Enfermagem em Nefrologia. Mestranda em Enfermagem da Universidade da Integração Internacional da Lusofonia Afro-Brasileira (UNILAB), Redenção-Ceará, Brasil. Orcid: https://orcid.org/0000-0002-0134-0694
} 


\section{INTRODUÇÃO}

Organizações e pesquisadores do mundo todo estão preocupados com a disseminação desse novo coronavírus denominado SARS-CoV-2, vírus responsável pelo surto da nova doença de coronavírus 2019 (COVID-19), detectada no dia 31 de dezembro de 2019 em Wuhan, na China ${ }^{(1)}$.

Embora as manifestações clínicas ocasionadas pela SARS-Cov-2 sejam semelhantes a uma Síndrome Gripal e um grande percentual de pessoas manifestem sintomas leves, merecem especial atenção as pessoas idosas, aquelas com comorbidades (doenças cardiovasculares, diabetes mellitus, asma, doença pulmonar obstrutiva crônica), gestantes e as mulheres até 45 dias pós-parto. Todas essas condições elevam as chances para a ocorrência das formas mais graves da doença causada pelo vírus SARS-CoV-2, a COVID-19. Outras complicações evidenciadas com maior incidência são a pneumonia, Síndrome Respiratória Aguda Grave (SRAG), insuficiência renal, sepse, miocardiopatias e aumento da atividade de coagulação, que favorece a ocorrência de eventos trombóticos ${ }^{(2)}$.

Alguns pacientes, por sua vez, cursam com um quadro grave da doença, necessitando em grande parte dos casos de internação em Unidade de Terapia Intensiva (UTI). Essa condição clínica se torna um risco particular para que esse sujeito seja acometido por um tromboembolismo pulmonar, o que torna fundamentalmente importante considerar os demais fatores de risco associados como o repouso prolongado, a principal enfermidade, a idade $\mathrm{e}$ as comorbidades, a fim de realizar uma profilaxia anticoagulante ${ }^{(3)}$.

Foi demonstrado consistentemente que o SARS-CoV-2 ocasiona uma tempestade de citocinas, as quais levam a ativação da cascata de coagulação que geram os fenômenos trombóticos, dentre eles a Coagulação Intravascular Disseminada $(\mathrm{CID})^{(4,5)}$. A CID é secundária às infecções graves, classicamente relacionada às infecções bacterianas, malária, febres hemorrágicas associadas aos vírus da dengue e do ebola, e agora também está relacionada à SARS-CoV-2. Um estudo detectou concomitância de isquemia (acro-isquemia) com o aparecimento de dispneia ${ }^{(6)}$. Essa observação levou aos pesquisadores a considerar a hipótese de insuficiência respiratória, em virtude da extensa obstrução capilar pulmonar por trombos, e que a CID desempenha um importante papel na hipoxemia, bem como nos resultados de morbidade e mortalidade dos pacientes internados em UTI com COVID-19 ${ }^{(6)}$.

Por se tratar de uma doença pandêmica, várias questões de pesquisa foram e vão surgindo à medida que a doença avança e se manifesta em diferentes povos do mundo. Pesquisas vêm sendo realizadas e já auxiliaram a esclarecer algumas temáticas como o genoma do vírus, as principais formas de transmissão do vírus e a prevenção da 
doença, a busca por medicamentos eficazes e seguros para o tratamento e a formulação e teste de vacinas. Entretanto, ainda são necessárias muitas pesquisas para aprofundar as lacunas acima elencadas.

A partir disso, surgiram os seguintes questionamentos: a) Qual é a incidência de eventos trombóticos em pacientes com SARSCoV-2 no mundo? b) Quais são os eventos trombóticos mais comuns em pacientes com SARS-CoV-2? c) Há diferença da incidência desses eventos trombóticos no tocante ao sexo, faixa etária e presença prévia de comorbidades? d) Quais as manifestações clínicas e laboratoriais mais comuns dos eventos trombóticos em pacientes com SARSCoV-2? e) Há diminuição da morbidade e mortalidade em pacientes com SARS-CoV-2 tratados com terapia anticoagulante e/ou protetor cardiovascular?

Diante dos fatos supracitados, o presente estudo tem por objetivo apresentar o conhecimento produzido sobre eventos trombóticos e sua relação com a infecção pela SARS-CoV-2. Tal trabalho é relevante, ao passo que, o SARS-CoV-2 é um vírus recente que ocasiona uma doença de fácil disseminação, com um número expressivo de mortes na população mundial e importantes e graves complicações, como os eventos trombóticos. Assim, são necessários que mais estudos sejam desenvolvidos para elucidar as questões relacionadas aos eventos trombóticos nos pacientes com COVID-19. Sua relevância se dá ainda porque apresenta uma síntese de muitos trabalhos que foram publicados sobre a temática COVID-19, munindo de informações relevantes os profissionais enfermeiros e os demais compõem as equipes de saúde interdisciplinares.

\section{MÉTODOS}

Para o alcance do objetivo, os autores deste estudo optaram pelo método da revisão integrativa da literatura, visto que reúne e sintetiza resultados de pesquisas sobre um delimitado tema, de maneira sistemática e organizada, contribuindo com o aprofundamento do conhecimento do tema investigado $^{(7)}$. As etapas da revisão integrativa da literatura foram: identificação da questão de pesquisa e objetivo do estudo, busca da literatura, avaliação dos dados, análise dos dados e apresentação ${ }^{(8)}$.

\section{Identificação da questão de pesquisa e do objetivo da revisão}

O tema de interesse foi: coagulopatias e eventos trombóticos relacionados com a infecção por SARS-CoV2. Com vistas a contemplar esse tema, o objetivo da revisão integrativa da literatura foi determinado como: apresentar o conhecimento produzido sobre os eventos trombóticos e a sua relação com a infecção por SARS-CoV-2.

Conforme já mencionado, as questões de pesquisa foram: a) Qual é a incidência de eventos trombóticos em 
pacientes com SARS-CoV-2 no mundo? b) Quais são os eventos trombóticos mais comuns em pacientes com SARS-CoV-2? c) Há diferença da incidência desses eventos trombóticos no tocante ao sexo, faixa etária e presença prévia de comorbidades? d) Quais as manifestações clínicas e laboratoriais mais comuns dos eventos trombóticos em pacientes com SARS-CoV-2? e) Há diminuição da morbidade e mortalidade em pacientes com SARS-CoV-2 tratados com terapia anticoagulante e/ou protetor cardiovascular?

\section{Busca na literatura}

As bibliotecas, bases e banco de dados selecionadas para a realização da busca bibliográfica foram: Biblioteca Virtual em Saúde, a qual contém as bases de dados LILACS, BDENF, WHO - IRIS, PAHO-IRIS e o diretório de revistas SCIELO; o portal da Pubmed: serviço da U.S. National Library of Medicine, contém a base de dados Medical Literature Analysis and Retrieval System Online (Medline); portal Cochrane e o buscador acadêmico Science Direct: uma das maiores fontes de base de dados de pesquisas da área da saúde, acessada pelo portal da Elsevier.

Com a utilização dessas diversas bases de dados, portais e bibliotecas, o âmbito da pesquisa foi ampliado para que dessa forma se pudessem minimizar possíveis vieses. Para o levantamento dos estudos contidos nestas bases de dados, bibliotecas e portais, realizou-se, inicialmente, a identificação dos descritores de cada base.

No tocante à biblioteca/base de dados/portais Pubmed, Science Direct, $e$ Cochrane, foi adotado o vocabulário $\mathrm{MeSH}$ Medical Subject Headings of U.S. National Library of Medicine - em língua inglesa, cujo descritores foram "coronavírus", "SARS" and "thrombus". Para a Biblioteca Virtual de Saúde foi utilizado o vocabulário estruturado DeCS - Descritores em Ciências da Saúde com os descritores "Vírus da SARS" and "Transtornos da Coagulação Sanguínea" e suas respectivas sinônimas em inglês e espanhol.

Destacamos que inicialmente foi realizada a busca com os descritores controlados em cada base de dados. Mas, em virtude do número pequeno de estudos encontrados, os descritores foram adotados como palavras-chave, ampliando o escopo da pesquisa. Os critérios de inclusão estabelecidos para os estudos foram: a) estudos completos que abordem a temática de coagulopatias e eventos trombóticos relacionados à infecção por SARS-CoV-2 b) estudos que respondam há pelo menos uma das questões de pesquisa.

A busca bibliográfica ocorreu no mês de maio de 2020 e foi atualizada no dia 28 de junho de 2020. Cada base de dados/biblioteca virtual/portal/buscador acadêmico foi acessada em um único dia, com gravação da página de busca. A seleção dos estudos foi realizada nos dias subsequentes, por meio da 
leitura e do título do resumo. Quando necessário, o texto completo foi acessado para realizar a leitura, obedecendo aos critérios de inclusão e exclusão estabelecidos.
Na Figura 1 será apresentado um fluxograma com a descrição completa do processo de seleção dos trabalhos incluídos na revisão integrativa da literatura.

Figura 1 - Fluxograma de seleção dos trabalhos identificados a partir das bases de dados selecionadas para esta revisão integrativa.

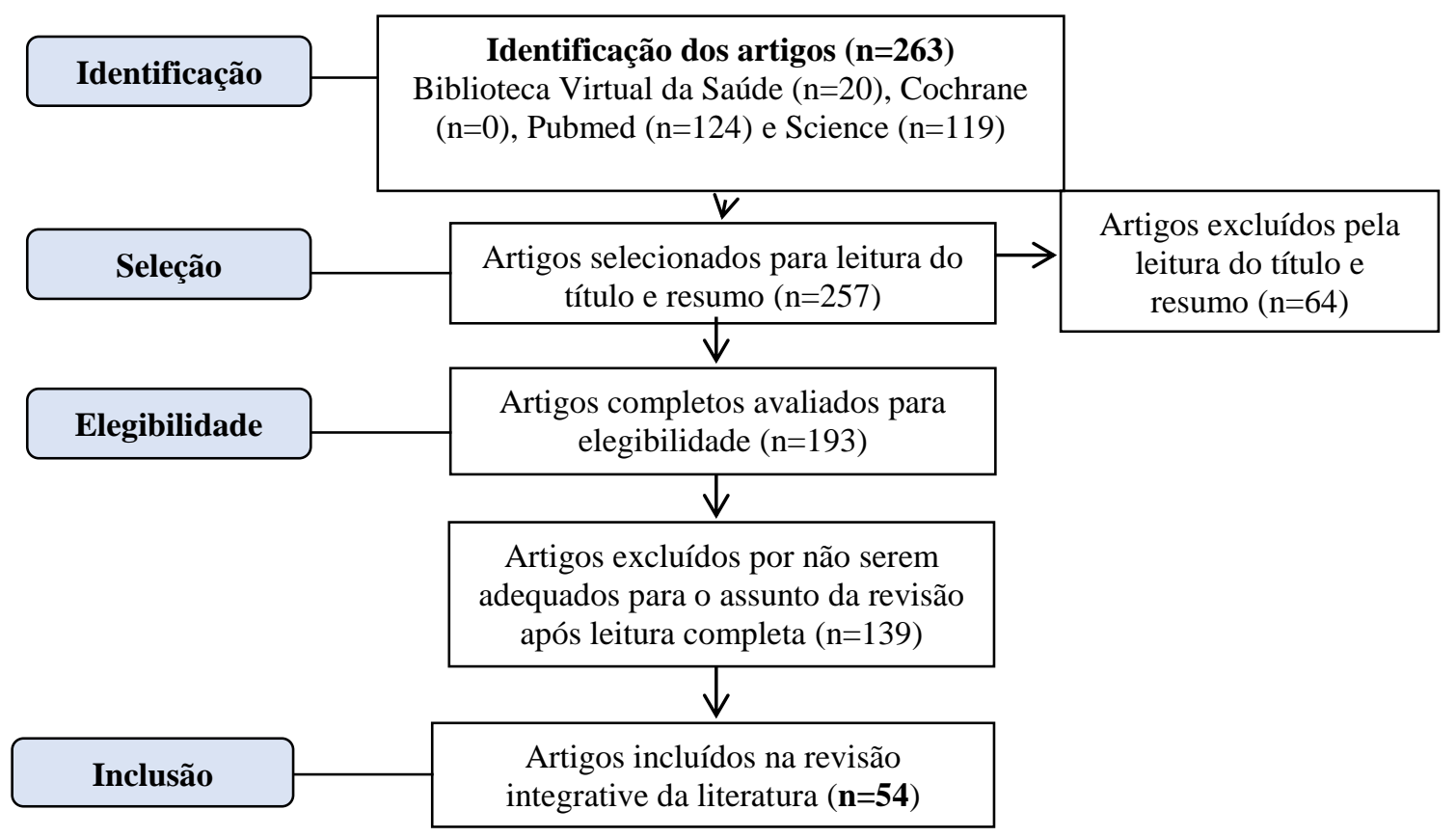

Fonte: Os autores (2020).

\section{Avaliação dos dados - foco na qualidade metodológica}

A avaliação dos estudos selecionados foi realizada em junho de 2020, utilizando uma classificação dos estudos segundo as forças de evidências ${ }^{(9)}$.

\section{Análise dos dados - divisão, exposição, comparação e apresentação dos dados}

Como as etapas 4 e 5 da Revisão Integrativa da Literatura são semelhantes, estas foram apresentadas em única seção. Todos os estudos selecionados foram sintetizados segundo algumas características: identificação, descrição metodológica, resultados encontrados e os níveis de evidência dos trabalhos.

Os dados foram apresentados na forma qualitativa, por meio de divisão e separação por categorias temáticas: incidência dos eventos trombóticos em pacientes com SARS-CoV-2 de acordo com as características sociodemográficas e clínicas; os eventos trombóticos mais comuns em pacientes com infecção por SARS-CoV-2 e as suas manifestações clínicas e laboratoriais; e 
os resultados dos tratamentos utilizados nessa situação clínica.

\section{Aspectos éticos}

Em relação aos aspectos éticos não houve a necessidade de envio do projeto ao Comitê de Ética em Pesquisa da Universidade da Integração Internacional da Lusofonia
Afro-Brasileira, de acordo com a Resolução $466 / 2012^{(10)}$, por se tratar de um estudo de revisão integrativa.

\section{RESULTADOS}

Em relação à avaliação da qualidade metodológica, os níveis de evidência ${ }^{(9)}$ dos trabalhos são apresentados na Tabela 1 .

Tabela 1 - Descrição da avaliação da qualidade metodológica dos artigos incluídos nesta revisão integrativa.

\begin{tabular}{c|c|c}
\hline Níveis de Evidência & $\begin{array}{c}\text { Frequência absoluta de } \\
\text { estudos }\end{array}$ & $\begin{array}{c}\text { Frequência relativa de } \\
\text { estudos }\end{array}$ \\
\hline 1 & $11^{(41)}$ & $2,0 \%$ \\
2 & 0 & $0,0 \%$ \\
3 & 0 & $0,0 \%$ \\
4 & $6^{(19,28,32,36,37,62)}$ & $11,1 \%$ \\
5 & $20^{(11,13,22,23,25,27,29,30,33-35,43-}$ & $37,0 \%$ \\
4 & $46,49,52,55,68,70)$ & $31,4 \%$ \\
7 & $17^{(15,20,21,24,26,40,42,47,48,53,54,56-}$ & $18,5 \%$ \\
\hline
\end{tabular}

Fonte: Os autores (2020).

Pela análise da Tabela 1, a maior parte dos trabalhos incluídos nessa revisão são provenientes de outras revisões, estudos descritivos e relatos de casos. Entretanto, é oportuno considerar que no momento atual, de emergência em saúde pública mundial, informações em saúde são relevantes, porque permitem a troca de informações entre os profissionais de saúde, a atualização constante do conhecimento científico, auxiliam a encontrar questões de pesquisa advindas de estudos de caso, estudos descritivos e estudos de revisão, às quais exigem estudos com delineamentos metodológicos mais robustos, e permitem também a orientação dos profissionais de saúde, que cuidam de pessoas com COVID-19, a tomar boas decisões, embasadas em evidências científicas.

Os resultados foram apresentados de acordo com as seguintes categorias temáticas: incidência dos eventos trombóticos em pacientes com SARS-CoV-2 de acordo com as características sociodemográficas e clínicas; os eventos trombóticos mais comuns em pacientes com infecção por SARS-CoV-2 e as suas manifestações clínicas e laboratoriais; e os resultados dos tratamentos utilizados nessa situação clínica. 
Incidência dos eventos trombóticos em pacientes com SARS-CoV-2 de acordo com as características sociodemográficas e clínicas

Evidências de parâmetros anormais de coagulação associados à COVID-19 apareceram no início de relatórios da China $^{(11)}$. Estudo desenvolvido com os 99 primeiros pacientes hospitalizados em Wuhan $^{(12)}$ encontrou que em $36 \%$ dos pacientes apresentaram D-dímero elevado, aumento dos biomarcadores inflamatórios, como a inteleucina-6, volume de hemossedimentação e proteína C-reativa. A trombocitopenia esteve presente em apenas $12 \%$ dos pacientes e quatro morreram de choque séptico $^{(12)}$.

Vários autores ${ }^{(13-17)}$ referenciaram o estudo realizado por Tang et al. ${ }^{(18)}$ sobre as coagulopatias e a COVID-19. Tal pesquisa ${ }^{(18)}$ avaliou 183 pacientes com SARS-CoV-2, entre os quais, 21 pacientes $(11,5 \%)$ morreram. Entre os 21 pacientes que morreram, $71 \%$ deles cumpriram os critérios da Sociedade Internacional de Trombose e Homeostasia para Coagulação Intravascular Disseminada, em comparação, com $0,6 \%$ dos pacientes sobreviventes.

Em âmbito mundial ainda não há estudos com base populacionais ou multicêntricos sobre a prevalência dos eventos trombóticos em pessoas com SARSCoV-2.

Várias pesquisas ${ }^{(13,19-35)}$ apontam que grande parte dos eventos trombóticos relacionados à SARS-CoV-2 ocorrem em pessoas idosas, do sexo masculino, com doenças crônicas pré-existentes como as cardiovasculares, cerebrovasculares, pulmonares, câncer, hepatopatias, nefropatias, obesidade e diabetes mellitus.

Um trabalho( ${ }^{(36)}$ sobre achados de autópsia e tromboembolismo venoso em pacientes com COVID-19 acrescentam além das doenças já citadas, a presença de doenças neurodegenerativas como fator de risco para as complicações trombóticas em pacientes com SARS-CoV-2. Um estudo de coorte italiano $^{(37)}$ observou que os valores de fibrinogênio pareciam ser maiores nas mulheres afetadas pela COVID-19 em comparação ao grupo controle. Uma tendência não significativa de aumento dos níveis de fibrinogênio foi demonstrada em pacientes do sexo masculino. Os pacientes com SARS e COVID-19 tendem a apresentar níveis significativos de fibrinogênio em comparação aos que não tem SARS, e os níveis de D-dímero mostraram uma tendência crescente não significativa.

Diretrizes sobre a prevenção e o tratamento do tromboembolismo recomendam a avalição do risco de tromboembolismo venoso baseado em muitas condições clínicas diferentes, tais como: idade acima de 40 anos, três dias acamado, confirmação laboratorial de COVID-19 combinado com um dos seguintes fatores de risco: presença de sepse, presença de doença respiratória aguda grave, insuficiência cardíaca, obesidade, história de 
tromboembolismo venoso, exacerbação aguda da doença pulmonar obstrutiva aguda, acidente vascular cerebral agudo, síndrome coronariana aguda, veias varicosas nos membros inferiores, câncer, doença inflamatória intestinal e insuficiência renal crônica $^{(38)}$.

Pesquisadores $^{(13,38-39)}$ afirmam que as mulheres grávidas e aquelas no período pósparto com COVID-19 têm mais chances de desenvolver tromboembolismo venoso. Este risco é potencializado por idade acima de 35 anos, história de tromboembolismo venoso, pré-eclâmpsia, crescimento intrauterino retardado, trombofilia genética, transfusão sanguina, infecções pós-parto, Lúpus Eritematoso Sistêmico, doenças cardíacas, obesidade, múltiplas gestações e hemorragias pós-parto ${ }^{(38-39)}$.

Um ponto de interesse na pandemia da COVID-19 tem sido as diferenças no padrão de mortalidade em jovens. Alguns jovens têm pior prognóstico, mas sem fatores de risco predisponentes e na Itália foram observadas altas taxas de mortalidade em pessoas mais jovens ${ }^{(40)}$. Uma das explicações para isso pode ser em decorrência de mutações na cascata de coagulação ou em fatores inibidores do complemento, como o fator $\mathrm{H}$ do complemento, que parece existir em $1 \%$ da população italiana ${ }^{(40)}$.

Entretanto, estudo de revisão sistemática $^{(41)}$ com metanálise sobre o impacto da doença cerebrovascular e cardiovascular na gravidade e mortalidade da
COVID-19 encontrou que tais doenças foram associadas ao aumento da mortalidade por COVID-19, mas o gênero, a idade, a hipertensão arterial, o diabetes mellitus e as condições respiratórias não influenciaram essa associação.

\section{Os eventos trombóticos mais comuns em pacientes com infecção por SARS-CoV-2 e as suas manifestações clínicas e laboratoriais}

O tromboembolismo relacionado ao vírus da SARS-CoV-2 pode ser observado nos vasos pulmonares, além de serem observados casos de pneumonia fibrinosa e organizadora. Assim, a lesão pulmonar de pequenos vasos sanguíneos é uma manifestação comum dos pulmões de pacientes com morte por COVID 19, sendo observada a trombose microvascular nos pulmões e tromboembolismo com infarto hemorrágico em vasos maiores. Na COVID-19 é observada claramente, na manifestação pulmonar, a trombose microvascular ${ }^{(21,24-25,28,30-34,36,42-46)}$.

Vários pesquisadores ${ }^{(11-12,17,25-28,33-}$ 34,37,45,47-53) descreveram que além do tromboembolismo pulmonar, o acidente vascular cerebral, o tromboembolismo venoso, o infarto agudo do miocárdio, o trombo do seio venoso cerebral, a coagulação intravascular disseminada e as coagulopatias induzidas por sepse são manifestações de eventos trombóticos em pacientes com SARSCoV-2.

Outros estudos ${ }^{(19,28,54)}$ relataram que a trombose de pequenos vasos levou à 
isquemia intestinal, colestase e distensão da vesícula biliar e infarto agudo de órgãos como baço, fígado e rins em pessoas com COVID19. Uma pesquisa ${ }^{(36)}$ também descreveu, com base em resultados de autópsia, que os êmbolos pulmonares são decorrentes de trombose venosa profunda de membros inferiores e que achados como trombose no plexo venoso prostático são também presentes em pessoas com infecção por SARS-CoV-2.

Em relação às complicações cardiovasculares relacionadas à COVID-19, estas envolvem a insuficiência cardíaca, miocardite, pericardite, vasculite e arritmias cardíacas. A presença de microangiopatia e microtrombos também pode predispor $\mathrm{o}$ paciente a micro-infartos em múltiplos órgãos, como fígado, coração ou rim, exacerbando ainda mais o estado de lesão e falência múltiplas de órgãos $(29,50-51,55)$.

Dois trabalhos descreveram eventos trombóticos nos capilares cutâneos, os quais geram a lesão capilar necrosante e que tem como manifestações clínicas erupções reticuladas levemente purpúricas no peito, pernas e braços, consistentes com livedo racemoso em alguns dos $\operatorname{casos}^{(56-57)}$. A explicação para essa manifestação é que como as células endoteliais também expressam o gene ACE2, a SARS-CoV-2 pode causar adicionalmente disfunção endotelial direta após a ligação à ECA2, levando a trombose subsequente ${ }^{(15)}$.

Outra pesquisa ${ }^{(40)}$ desenvolvida sobre a análise da deposição do vírus SARS-
CoV-2 em cinco placentas relatou que houve a trombose em vasos maiores da circulação fetal, trombose de Frank fetal em três casos, enquanto nos outros dois casos foi confirmada trombose vascular confinada às vilosidades do tronco, gerando uma má perfusão fetal por trombose em vasos da placenta.

As manifestações clínicas e laboratoriais dos eventos trombóticos em pessoas com SARS-CoV-2 estão correlacionadas com os sinais e sintomas específicos da trombose venosa central ${ }^{(26,48)}$, do acidente vascular cerebral ${ }^{(41,47,49,53)}$ do infarto agudo do miocárdio ${ }^{(13,21,29,41)}$, da trombose pulmonar ${ }^{(28,31,33,45,58)}$, trombose venosa periférica profunda ${ }^{(20)}$, coagulação intravascular disseminada $a^{(14,45,58)}$ e da insuficiência renal aguda ${ }^{(53,59-60)}$.

Pesquisa sobre achados de anormalidades intestinais ${ }^{(19)}$ evidenciou que na tomografia computadorizada de pacientes da UTI podem ser observadas anormalidades na parede intestinal. Presença de sintomas do trato gastrointestinal como náusea, vômito, diarreia ou dor abdominal e o espessamento da parede intestinal, pneumatose e gás venoso portal devido isquemia mesentérica em pacientes críticos acometidos pela SARSCoV-2.

No tocante aos exames laboratoriais, vários estudos ${ }^{(11,16,17,24,30,46,49,61)} \quad$ citaram valores elevados do D-dímero como fator predisponente para a ocorrência dos eventos trombóticos em pacientes com COVID-19. 


\section{Resultados dos tratamentos utilizados nessa situação clínica}

Os relatórios atuais demonstram benefícios das heparinas na profilaxia dos eventos trombóticos em pacientes com COVID-19. Alguns estudos apontam que profissionais têm adotado tratamentos com heparina de baixo peso molecular (enoxaparina) ou heparina não fracionada. $\mathrm{O}$ sucesso do tratamento parece depender da idade e dos valores de D-dímero do paciente, pois quando o valor desse marcador excede seis vezes o limite superior normal, a mortalidade se torna menor com o tratamento $^{(22,55)}$.

No entanto, os pacientes da UTI com COVID-19 devem fazer uso de doses mais altas de heparina de baixo peso molecular, pois foi demonstrado que eles desenvolvem tromboembolismo pulmonar mesmo com o tratamento profilático. Assim, tanto pacientes admitidos na UTI quanto em enfermarias, mesmo recebendo heparina de baixo peso molecular, estão desenvolvendo trombose venosa profunda ou embolia pulmonar, o que reforça que as doses de heparina devem ser dobradas nesse contexto clínico (11,16,18,20,22$23,25,27,30,33,38,43,45,62-63)$

Um outro estudo sobre embolia pulmonar em pacientes com COVID-19 acrescentou que houve uma melhora no efeito antitrombótico quando houve a mudança da enoxaparina para rivaroxabana ${ }^{(24)}$. Pesquisadores $^{(25)}$ acrescentam que para pacientes com acidente vascular cerebral e a
COVID-19 parece ser razoável tratar com tPA. Além disso, bloqueadores do receptor AT1, como a losartana, pode ter efeito protetor em pacientes com acidente vascular cerebral.

Estudiosos (16,18,34,64) relatam os resultados sobre o uso da imunoglobulina intravenosa (IGIV) e da terapia anticoagulante com heparina de baixo peso molecular o mais cedo possível, principalmente quando o número de linfócitos $\mathrm{T}$ e $\mathrm{B}$ circulantes diminui e citocinas inflamatórias e D-Dímero aumentam anormalmente. Embora a imunoglobulina intravenosa tenha mostrado eficácia no tratamento de pacientes com influenza e SARS são necessários mais dados clínicos, a fim de determinar que a imunoglobulina possui uma eficácia significativa em pacientes com COVID-19.

É oportuno salientar que estudos préclínicos $^{(65-67)}$ documentam os efeitos antitrombóticos da suplementação de vitamina D. Pesquisadores também ressaltam que a maior parte dos estudos de intervenção não mostram claramente os efeitos protetores da suplementação da vitamina D na prevenção de eventos cardiovasculares. Todavia, a maioria dessas pesquisas não avaliaram pessoas com deficiências severas de vitamina D (baseline $)^{(67-68)}$. A deficiência de vitamina D é um problema prevalente em todo o mundo, $7 \%$ da população mundial tem deficiência severa e cerca de $40 \%$ tem níveis limítrofes de vitamina D no corpo ${ }^{(66)}$. 
Em relação à proteção cardiovascular, poucos trabalhos ${ }^{(29,51)}$ descreveram que em pacientes com COVID19 e com lesão cardíaca, devem ser rigorosamente monitorizados em relação ao aumento das enzimas cardíacas - os marcadores de estresse cardíaco e que a administração de agentes cardioprotetores se faz necessária.

\section{DISCUSSÃO}

Entre as explicações sobre a formação de microtrombos com lesões em diversos órgãos-alvo temos que os vírus da SARS-CoV-2 possui uma extensa distribuição tissular ocasionando a liberação de um alto número de citocinas pró-inflamatórias (tempestade de citocinas), promovendo uma síndrome de resposta inflamatória sistêmica com impacto importante no sistema hematopoiético e na hemostasia, levando ao infarto de órgãos como os pulmões, coração, fígado e rins ${ }^{(14,50,69) \text {. }}$

É oportuno destacar que as alterações específicas da hemostasia já estão presentes em estágio inicial da infecção pela SARSCoV-2(11,18,37). A coagulopatia associada à COVID-19 por resposta inflamatória sistêmica aguda é caracterizada por uma elevação de vários marcadores de coagulação, por exemplo, D-dímero, produto de degradação da fibrina ou fibrinogênio, aumento concomitante de marcadores inflamatórios (por exemplo, proteína C reativa) e trombocitopenia leve $\mathrm{e}^{(49,61)}$.
Entre as hipóteses que justificam o aumento da incidência dos eventos trombóticos em pessoas mais velhas e com doenças crônicas pré-existentes estão: a) tendência dessas pessoas terem um valor D dímero mais alto $^{(16,18,34)}$; b) a utilização de medicamentos anti-hipertensivos, como os inibidores da ECA e os bloqueadores dos receptores de angiotensina II , que aumentam os níveis de expressão do gene ECA2, e favorecem a replicação do vírus SARS-CoV$2^{(30)}$; c) alteração do sistema reninaangiotensina-aldosterona está intrinsecamente ligado à cascata de coagulação e pode agravar o processo de imunotrombose, impulsionando ainda mais a formação de microtrombos na COVID-19(70).

Há evidências fortes que existem a oclusão e a formação de microtrombos em pequenos vasos pulmonares em pacientes críticos com COVID-19 e, que, portanto, a aplicação precoce de terapia anticoagulante nesses pacientes é importante para melhorar o prognóstico $^{(18)}$. Todo paciente com risco de trombose venosa profunda internado em UTI deve receber algum tratamento preventivo (farmacológico, mecânico ou os dois), pois a prevenção é essencial, desde que segura e efetiva. A equipe de enfermagem é primordial nesse processo, desde a administração dos fármacos prescritos até a realização de medidas não farmacológicas que contribuam como parte desse tratamento preventivo descrito. 
Os enfermeiros e os demais profissionais devem estar atentos ainda aos sinais e sintomas de eventos trombóticos manifestados pelos pacientes com COVID-19, porque comprovadamente é uma doença que pode gerar tais complicações, as quais carecem de tomadas de decisão clínica efetivas em tempo oportuno. Tais complicações repercutem no perfil de morbimortalidade, conforme descrito. Essa reponsabilidade se aplica ainda mais à equipe de enfermagem por serem os profissionais que mais prestam cuidados assistenciais diretos aos clientes.

A recomendação para os enfermeiros e demais profissionais de saúde que prestam cuidados de enfermagem a pacientes críticos ou semicríticos nos hospitais de campanha e de referência para COVID-19, é que monitorizem de perto os pacientes enfermos. É formidável que se atentem no que se refere aos sinais e sintomas de eventos trombóticos, aos marcadores inflamatórios e ao estado de hipercoagulabilidade que eles apresentam. Ademais, que possam reconhecer precocemente o risco elevado de trombose nesses pacientes, principalmente nos indivíduos idosos com comorbidades, com destaque para as cardiovasculares, pulmonares, endócrinas - diabetes mellitus e metabólicas - obesidade, para a implementação precoce eficaz de terapia anticoagulante.

Algumas questões de pesquisa elaboradas não foram respondidas, como a incidência dos eventos trombóticos em pessoas com SARS-CoV-2 em âmbito mundial e os efeitos de medicamentos protetores cardiovasculares na diminuição da morbidade e mortalidade. O apelo é que os pesquisadores das mais diversas áreas da saúde possam buscar respondê-las ao longo do tempo para que prontamente se possam publicar os seus achados.

Esse estudo apresenta as seguintes implicações: a) traz uma síntese aprofundada e atualizada do conhecimento produzido por pesquisadores do mundo todo sobre complicações graves advindas do vírus da SARS-CoV-2, tais como os eventos trombóticos; b) pode auxiliar a tomada de decisão de médicos e enfermeiros para o monitoramento e tratamento de pessoas com COVID-19 que estão sob os seus cuidados; c) descreve lacunas de conhecimento e questões de pesquisa que precisam ser investigadas em outros trabalhos para contribuir com a formulação do conhecimento na área; d) no contexto dessa pandemia, estudos que envolvem a temática da infecção por SARSCoV-2 e a COVID 19 são muito importantes, independente das abordagens, porque é uma doença nova e o conhecimento que vem sendo produzido e divulgado têm diminuído os graves impactos ocasionados pela infecção pelo vírus da SARS-CoV-2.

Muitas questões apontadas nesta revisão integrativa precisam ser melhores investigadas para maiores esclarecimentos, tais como a a incidência dos eventos 
trombóticos relacionados à infecção pelo vírus da SARS-CoV-2 nos diferentes continentes; e se existem fatores protetores efetivos comprovados (clínicos, laboratoriais, farmacológicos) que diminuiriam as chances de desenvolvimento dos eventos trombóticos em pessoas infectadas pela SARS-CoV-2; e quais as ferramentas utilizadas pela equipe de enfermagem comprovadamente efetivas para a redução na incidência desse agravo para essa população.

Outros questionamentos também assinalados, os quais também requerem mais investigação, foram se a suplementação de vitamina $\mathrm{D}$ é eficaz para a prevenção de eventos trombóticos em pessoas com COVID19; o comparativo entre os métodos não farmacológicos e farmacológicos de prevenção de trombose venosa em pessoas com COVID 19; e os estudos de acompanhamento de pessoas que tiveram a COVID-19, para averiguar a incidência de eventos trombóticos, em fase posterior ou para a investigação de uma reincidência de novos eventos entre aqueles que manifestaram alguma doença trombótica na fase inicial da infecção pela SARS-CoV-2.

\section{CONSIDERAÇÕES FINAIS}

Os trabalhos incluídos nesta revisão apontam que existe uma relação entre a fisiopatologia da infecção por SARS-CoV-2 e os eventos trombóticos, por meio da tempestade citocinas e a cogulação intravascular disseminada. A literatura evidenciou ainda que as chances de ocorrência de eventos trombóticos na COVID-19 aumentam em pessoas mais idosas e com comorbidades associadas. Trouxe também que os eventos trombóticos nessa situação clínica mais descritos foram trombose pulmonar microvascular, tromboembolismo venoso de membros inferiores, síndrome coronariana aguda e acidente vascular cerebral.

A avaliação e acompanhamento de pacientes, principalmente os internados em UTI, no tocante ao seu estado inflamatório e os seus marcadores de hipercoagulabilidade, como D dímero, foram apresentadas como importantes para a detecção precoce e tomada de decisão para a administração de terapias anticoagulantes. A enfermagem é primordial para o acompanhamento e monitoramento desses pacientes, bem como para a rápida $\mathrm{e}$ efetiva tomada de decisão para estabilidade do quadro clínico de pacientes críticos até a chegada dos demais profissionais que constituem a equipe multiprofissional de cuidados intensivos.

Embora a incidência dos eventos trombóticos em pessoas com COVID-19 em âmbito mundial já esteja melhor descrita na literatura, ainda é necessário que se produzam mais estudos clínicos e epidemiológicos para melhor explicar essa relação. É importante ainda para que se possa determinar se de fato essa doença pode ser classificada como pertencente ao grupo de doenças vasculares. 
Os dados desse estudo poderão subsidiar mais pesquisas interdisciplinares nessa linha temática.

\section{REFERÊNCIAS}

1. World Health Organization. Coronavírus (COVID-19) Dashboard. Disponível em: https://covid19.who.int/ Acessed: 05 de maio de 2020.

2. Strabelli TMV, Uip DE. COVID-19 e o Coração. Arq Bras Cardiol 2020 Mar; 114(4). DOI: https://doi.org/10.36660/abc.20200209

3. Mascarello MG, Vanonni G, Indavere A, Waistein KM, Estrella ML, Rodriguez SG et al. Tromboembolismo de pulmón - sospecha clínica y correlación anatomopatológica. Medicina (B Aires) 2020; 80(2): 97-102.

4. Han H, Yang L, Liu R, Liu F, Wu KL, Li J et al. Prominent changes in blood coagulation of patients with SARSCoV- 2 infection. Clin Chem Lab Med 2020; 58(7):1116-1120. DOI: 10.1515 / cclm-2020-0188

5. Wu C, Chen X, Cai Y, Xia JA, Zhou X, $\mathrm{Xu}, \mathrm{S}$ et al. Risk Factors Associated With Acute Respiratory Distress Syndrome and Death in Patients With Coronavirus Disease 2019 Pneumonia in Wuhan, China. JAMA Intern Med 2020; e200994. DOI: 10.1001 / jamainternmed.2020.0994

6. Negri EM, Piloto BM, Morinaga LK, Jardim CVP, Lamy SAE, Ferreira MA et al. Heparin Therapy improving hipóxia in COVID-19 patients - a case series. MedRxiv 2020.

DOI: https://doi.org/10.1101/2020.04.15.20067017

7. Mendes KDS, Silveira RCCP, Galvão CM. Revisão integrativa: método de pesquisa para a incorporação de evidências na saúde e na enfermagem. Texto contexto - enferm 2008; 17(4):758-764.

DOI: https://doi.org/10.1590/S0104-

07072008000400018

8. Whittemore R, Knafl K. The integrative review: updated methodology. J Adv Nurs 2005; 52(5):546-553. DOI: https://doi.org/10.1111/j.1365-

2648.2005.03621.x
9. Melnyk BM, Fineout-Overholt E. Making the case for evidence-based practice. In: Melnyk BM, Fineout-Overholt E. Evidencebased practice in nursing \& healthcare - a guide to best practice. Philadelphia: Lippincot Williams\& Wilkins; 2005.

10. Brasil Resolução $N^{\circ} 466 / 12$. Diretrizes e Normas regulamentadoras das pesquisas que envolvem seres humanos. Brasília: Conselho Nacional de Saúde; 2012.

11. Connors JM, Levy JH. COVID-19 and Its Implications for Thrombosis and Anticoagulation. Blood 2020 Apr 27; 135(23): 2033-2040. DOI: https://doi.org/10.1182/blood.2020006000

12. Chen N, Zhou M, Dong X, Qu J, Gong F, Han $\mathrm{Y}$ et al. Epidemiological and clinical characteristics of 99 cases of 2019 novel coronavirus pneumonia in Wuhan, China: a descriptive study. Lancet 2020; 395:507-13. DOI: https://doi.org/10.1016/S01406736(20)30211-7

13.Bikdeli B, Madhavan MV, Jimenez D, Chuich T, Dreyfus I, Driggin E, et al. COVID-19 and Thrombotic or Thromboembolic Disease: Implications for Prevention, Antithrombotic Therapy, and Follow-up. Journal of the American College of Cardiology 2020. DOI: https://doi.org/10.1016/j.jacc.2020.04.031

14. Song JC, Wang G, Zhang W, Zhang Y, Li WQ, Zhou Z. Chinese expert consensus on diagnosis and treatment of coagulation dysfunction in COVID-19. Military Medical Research 2020;7(1). DOI: https://doi.org/10.1186/s40779-020-00247-7

15. Thomas C. Reply to: "A Dermatologic Manifestation of COVID-19: Transient Livedo Reticularis." Journal of the American Academy of Dermatology 2020. DOI: https://doi.org/10.1016/j.jaad.2020.04.164

16. Thachil J, Tang N, Gando S, Falanga A, Cattaneo M, Levi $\mathrm{M}$, et al. ISTH interim guidance on recognition and management of coagulopathy in COVID-19. J Thromb Haemost 2020.2 DOI: https://doi.org/10.1111/jth.14810

17. Vivas D, Roldán V, Esteve-Pastor M A, Roldán I, Tello-Montoliu A, Ruiz-Nodar J M, 
et all. Recomendaciones sobre el tratamiento antitrombótico durante la pandemia COVID19. Posicionamiento del Grupo de Trabajo de Trombosis Cardiovascular de la Sociedad Española de Cardiología. Revista Española de Cardiología 2020. DOI: https://doi.org/doi:10.1016/j.recesp.2020.04.0 06

18. Tang N, Li D, Wang X, Sun Z. Abnormal coagulation parameters are associated with poor prognosis in patients with novel coronavirus pneumonia. J Thromb Haemost 2020. DOI: https://doi.org/10.1111/jth.14768

19. Bhayana R, Som A, Li MD, Carey DE, Anderson MA, Blake MA, et al. Abdominal Imaging Findings in COVID-19: Preliminary Observations. Radiology 2020 May 11; 201908. DOI: https://doi.org/10.1148/radiol.2020201908

20. Bozzani A, Arici V, Franciscone MM, Danesino V, Cascina A, Ticozzelli G, et al. Severe Acute Respiratory Syndrome Coronavirus 2 Infection and the Upper Limb Deep Vein Thrombosis Risk. Ann Vasc Surg 2020 Apr 23; S0890-5096(20)30356-3. DOI: https://doi.org/10.1016/j.avsg.2020.04.037

21. Buja LM, Wolf D, Zhao B, Akkant B, McDonald M, Lelenwa L, et al. Emerging spectrum of cardiopulmonary pathology of the coronavirus disease 2019 (COVID-19): report of three autopsies from houston, texas and review of autopsy findings from other united states cities. J Card Fail 2020 Apr; PII: S1054-8807(20)30037-5.

DOI: https://doi.org/10.1016/j.carpath.2020.107233

22. Harenberg J, Favaloro E. COVID-19: Progression of Disease and Intravascular Coagulation - Present Status and Future Perspectives. Clin Chem Lab Med 2020 May 14; 58(7): 1029-1036. DOI: https://doi.org/10.1515/cclm-2020-0502

23. Giannis D, Ziogas IA, Gianni P. Coagulation Disorders in Coronavirus Infected Patients: COVID-19, SARS-CoV-1, MERS-CoV and Lessons From the Past. J Clin Virol 2020 Apr 9; 127: 104362. DOI: 10.1016/j.jcv.2020.104362.

24. Griffin DO, Jensen A, Khan M, Chin J, Chin K, Saad J, et al. Pulmonary Embolism and Increased Levels of d-Dimer in Patients with Coronavirus Disease. Emerg Infect Dis $2020 \quad$ Apr 29; 26(8). DOI: 10.3201/eid2608.201477.

25. Hess DC, Eldahshan W, Rutkowski E. COVID-19-Related Stroke. Transl Stroke Res 2020 May 7; 11(3): 322-325. DOI: 10.1007/s12975-020-00818-9.

26. Hughes C, Nichols T, Pike M, Subbe C, Elghenzai S. Cerebral Venous Sinus Thrombosis as a Presentation of COVID-19. Eur J Case Rep Intern Med 2020 Apr 29; 7(5): 001691. DOI: 10.12890/2020_001691.

27. Kollias A, Kyriakoulis KG, Dimakakos E, Poulakou G, Stergiou GS, Syrigos K. Thromboembolic Risk and Anticoagulant Therapy in COVID-19 Patients: Emerging Evidence and Call for Action. Br J Haematol 2020 May 4; 189(5): 846-847. DOI: 10.1111/bjh.16727.

28. Lax SF, Skok K, Zechner P, Kessler HH, Kaufmann N, Koelblinger C, et al. Pulmonary Arterial Thrombosis in COVID-19 With Fatal Outcome: Results From a Prospective, SingleCenter, Clinicopathologic Case Series. Ann Intern Med 2020 May 14; M20-2566. DOI: 10.7326/M20-2566.

29. Liu PP, Blet A, Smyth D, Li H. The Science Underlying COVID-19: Implications for the Cardiovascular System. Circulation 2020 Apr $15 . \quad$ DOI: 10.1161/CIRCULATIONAHA.120.047549.

30. Alijotas-Reig J, Esteve-Valverde E, Belizna C, Selva-O'Callaghan A, Pardos-Gea $\mathrm{J}$, Quintana A, et al. Immunomodulatory therapy for the management of severe COVID-19. Beyond the anti-viral therapy: A comprehensive review. Autoimmun Rev 2020 Apr; PII: S1568-9972(20)30131-2. DOI: https://doi.org/10.1016/j.autrev.2020.102569

31. Saba L, Sverzellati N. Is COVID Evolution Due to Occurrence of Pulmonary Vascular Thrombosis? J Thorac Imaging 2020 Apr 28; 10.1097/RTI.0000000000000530. DOI: 10.1097/RTI.0000000000000530.

32 Spiezia L, Boscolo A, Poletto F, Cerruti L, Tiberio I, Campello E, et al. COVID-19Related Severe Hypercoagulability in Patients Admitted to Intensive Care Unit for Acute 
Respiratory Failure. Thromb Haemost 2020 Apr 21; 120(6): 998-1000. DOI: 10.1055/s0040-1710018.

33. Tal S, Spectre G, Kornowski R, Perl L. Venous Thromboembolism Complicated with COVID-19: What Do We Know So Far? Acta Haematol 2020 May 12; 1-8. DOI: $10.1159 / 000508233$.

34. Thachil J, Srivastava A. SARS-2 Coronavirus-Associated Hemostatic Lung Abnormality in COVID-19: Is It Pulmonary Thrombosis or Pulmonary Embolism?. Semin Thromb Hemost 2020 May 12. DOI: 10.1055/s-0040-1712155.

35. Verdecchia P, Cavallini C, Spanevello A, Angeli F. The Pivotal Link Between ACE2 Deficiency and SARS-CoV-2 Infection. Eur J Intern Med 2020 Apr 20; 76: 14-20. DOI: 10.1016/j.ejim.2020.04.037.

36. Wichmann D, Sperhake JP, Lütgehetmann M, Steurer S, Edler C, Heinemann A, et al. Autopsy Findings and Venous Thromboembolism in Patients With COVID19: A Prospective Cohort Study. Ann Intern Med 2020 May 6; M20-2003. DOI: 10.7326/M20-2003.

37. Di Micco P, Russo V, Carannante N, Imparato $\mathrm{M}$, Rodolfi $\mathrm{S}$, Cardillo $\mathrm{G}$, et al. Clotting Factors in COVID-19: Epidemiological Association and Prognostic Values in Different Clinical Presentations in an Italian Cohort. J Clin Med 2020 May 7; 9(5):1371. DOI: $10.3390 / \mathrm{jcm} 9051371$.

38 Zhai Z, Li C, Chen Y, Gerotziafas G, Zhang Z, Wan J, et al. Prevention and Treatment of Venous Thromboembolism Associated with Coronavirus Disease 2019 Infection: A Consensus Statement before Guidelines. Thromb Haemost. 2020;120(6):937-948. doi:10.1055/s-00401710019

39. Leffert L, Butwick A, Carvalho B, et al; members of the SOAP VTE Taskforce. The Society for Obstetric Anesthesia and Perinatology Consensus Statement on the anesthetic management of pregnant and postpartum women receiving thromboprophylaxis or higher dose anticoagulants.

2018;126(03):928-

Anesth

Analg

944.

40. Mulveya JJ, Magrob CM, Mab LX, Nuovoc GJ, Baergenb RN. Analysis of complement deposition and viral RNA in placentas of COVID-19 patients. Ann Diagn Pathol 2020; 46:151529. DOI: https://doi.org/10.1016/j.anndiagpath.2020.15 1529

41. Pranata R, Huang I, Lim MA, Wahjoepramono PEJ, July J. Impact of Cerebrovascular and Cardiovascular Diseases on Mortality and Severity of COVID-19Systematic Review, Meta-analysis, and Metaregression. Journal of Stroke and Cerebrovascular Diseases 2020. https://doi.org/10.1016/j.jstrokecerebrovasdis. 2020.104949

42. Audo A, Bonato V, Cavozza C, Maj G, Pistis G, Secco GG. Acute Pulmonary Embolism in SARS-CoV-2 Infection Treated with Surgical Embolectomy. Ann Thorac Surg 2020 Apr; PII: S0003-4975(20)30616-0. DOI:

https://doi.org/10.1016/j.athoracsur.2020.04.0 13

43. Tersalvi G, Vicenzi M, Calabretta D, Biasco L, Pedrazzini G, Winterton D. Elevated troponin in patients with Coronavirus Disease 2019 (COVID-19): possible mechanisms. J Card Fail 2020 Apr; PII: S1071-9164(20)30357-2 DOI: https://doi.org/10.1016/j.cardfail.2020.04.009

44. Zhang J, Xie B, Hashimoto K. Current status of potential therapeutic candidates for the COVID-19 crisis. Brain Behav Immun 2020 Apr; PII: S0889-1591(20)30589-4. DOI: https://doi.org/10.1016/j.bbi.2020.04.046

45. Whyte CS, Morrow GB, Mitchell JL, Chowdary P, Mutch NJ. Fibrinolytic Abnormalities in Acute Respiratory Distress Syndrome (ARDS) and Versatility of Thrombolytic Drugs to Treat COVID-19. J Thromb Haemost 2020 Apr 23. DOI: 10.1111/jth.14872.

46. Xie M, Chen Q. Insight into 2019 novel coronavirus - An updated interim review and lessons from SARS-CoV and MERS-CoV. Int 
J Infect Dis 2020 Mar; 94(2020): 119-24. DOI:

https://doi.org/10.1016/j.ijid.2020.03.071

47. Avula A, Nalleballe K, Narula N, Sapozhnikov S, Dandu V, Toom S, et al. COVID-19 presenting as stroke. Brain Behav Immun 2020 Apr; PII: S08891591(20)30685-1. DOI: https://doi.org/10.1016/j.bbi.2020.04.077

48. Dahl-Cruz F, Guevara-Dalrymple N, López-Hernández N. Cerebral Venous Thrombosis and SARS-CoV-2 Infection. Rev Neurol 2020 May 16; 70(10): 391-392. DOI: 10.33588/rn.7010.2020204.

49. Divani AA, Andalib S, Napoli SD, Lattanzi S, Hussain S, Biller $J$, et al. Coronavirus disease 2019 and stroke: clinical manifestations and pathophysiological insights. J Stroke Cerebrovasc Dis 2020 May; PII: $\quad$ S1052-3057(20)30347-5. DOI: https://doi.org/10.1016/j.jstrokecerebrovasdis. 2020.104941

50. Katal S, Balakrishnan S, Gholamrezanezhad A. Neuroimaging findings in COVID-19 and other coronavirus infections: a systematic review in 116 patients. AJNR Am. J. Neuroradiol 2020; PII: S0150-9861(20)30204-2.

Doi: https://doi.org/10.1016/j.neurad.2020.06.007

51. Mahajan K, Chandra KS. Cardiovascular comorbidities and complications associated with coronavirus disease 2019. Med J. Armed Forces India 2020 May; Doi: https://doi.org/10.1016/j.mjafi.2020.05.004

52. Pedicelli A, Valente I, Pilato F, Distefano M, Colosimo C. Stroke priorities during COVID-19 outbreak: acting both fast and safe. J Stroke Cerebrovasc Dis 2020 May; 104922.

DOI: https://doi.org/10.1016/j.jstrokecerebrovasdis. 2020.104922.

53. Wright FL, Vogler TO, Moore EE, Moore $\mathrm{HB}$, Wohlauer MV, Urban $\mathrm{S}$, et all. Fibrinolysis Shutdown Correlates to Thromboembolic Events in Severe COVID19 Infection. Journal of the American College of Surgeons. 2020. https://doi.org/10.1016/j.jamcollsurg.2020.05. 007
54. Barry O, Mekki A, Diffre C, Seror M, Hajjam MEL, Carlier RY. Arterial and venous abdominal thrombosis in a 79-yearold woman with COVID-19 pneumonia. Radiol Case Rep 2020 Apr; PII: S19300433(20)30158-8.

DOI: https://doi.org/10.1016/j.radcr.2020.04.055

55. Ganatra S, Dani SS, Shah S, Asnani S, Neilan TG, Lenihan D, et al. Management of Cardiovascular Disease During Coronavirus Disease (COVID-19) Pandemic. Trends Cardiovas Med 2020 June 5; 21:14. Doi: https://doi.org/10.1016/j.tcm.2020.05.004

56. Magro C, Mulvey JJ, Berlin D, Nuovo G, Salvatore S, Harp J, et al. Complement associated microvascular injury and thrombosis in the pathogenesis of severe COVID-19 infection: A report of five cases. Transl Res 2020. PII: S1931-5244(20)300700 .

DOI:

https://doi.org/10.1016/j.trs1.2020.04.007

57. Rebollar JCO. Manifestaciones cutaneas de la infeccion por covid 19. a proposito de un caso. Medicina Clínica. 2020. https://doi.org/10.1016/j.medcli.2020.04.002

58. Xing C, Li Q, Du H, Kang W, Lian J, Yuan L. Lung Ultrasound Findings in Patients With COVID-19 Pneumonia. Crit Care 2020 Apr 28; 24(1): 174. DOI: 10.1186/s13054020-02876-9.

59. Post A, Deurwaarder ESG, Bakker SJL, Haas RJ, Meurs M, Gansevoort RT, et al. Kidney Infarction in Patients With COVID19. Am J Kidney Dis. 2020; XX(XX):1- 5. Doi:

https://doi.org/10.1053/j.ajkd.2020.05.004

60. Selby NM, Forni LG, Laing CM, Horne KL , Evans RDr, Lucas BJ, et al. Covid-19 and acute kidney injury in hospital: summary of NICE guidelines. BMJ. 2020; 369: m1963. Published $2020 \quad$ May 26. doi:10.1136/bmj.m1963

61. Kaur P, Qaqa F, Ramahi A, Shamoon Y, Singhal M, Shamoon F, Maroules M, Singh B. Acute upper limb ischemia in a patient with COVID-19. Hematol Oncol Stem Cell Ther 2020 May; PII: S1658-3876(20)300960 .

DOI: https://doi.org/10.1016/j.hemonc.2020.05.001 
62. Helms J, Tacquard C, Severac F, LeonardLorant I, Ohana M, Delabranche X, et al. High risk of thrombosis in patients with severe SARS-CoV-2 infection: a multicenter prospective cohort study. Intensive Care Med 2020 Jun; 46(6): 1089-1098. DOI: 10.1007/s00134-020-06062-x.

63. Hirshberg A, Kern-Goldberger AR, D. Levine L, Pierce-Williams R, Short WR, Parry $\mathrm{S}$, et al. Care of critically ill pregnant patients with COVID-19: a case series. Am J Obstet Gynecol 2020 Apr; PII: S00029378(20)30515-9.

DOI: https://doi.org/10.1016/j.ajog.2020.04.029

64 Raucci F, Mansour AA, Casillo GM, Saviano A, Caso F, Scarpa R, et all. Interleukin-17A (IL-17A), a key molecule of innate and adaptive immunity, and its potential involvement in COVID-19-related thrombotic and vascular mechanisms. Autoimmunity Reviews. 2020. https://doi.org/10.1016/j.autrev.2020.102572

65. Gomez JMQ, Castillo ME, Bouillon R. Vitamin D Receptor stimulation to reduce Acute Respiratory DistressSyndrome (ARDS) in patients with Coronavirus SARS-CoV-2 infections. J Steroid Biochem 2020 May 4; PII: S0960-0760(20)30244-2. DOI: https://doi.org/10.1016/j.jsbmb.2020.105719

66. Bouillon R. Vitamin D status in Africa is worse than in other continents. Lancet Glob. Heal 2020; 8:e20-e21. DOI: 10.1016 / S2214109X (19) 30492-9

67. Bouillon R, Marcocci C, Carmeliet D, Bikle JH, White B, Dawson, P et al. Skeletal and extraskeletal actions of vitamin D: current evidence and outstanding questions. Endocr. Rev 2019; 40:1109-1151. DOI: 10.1210/er.2018-00126

68. Weir EK, Thenappan T, Bhargava M, Chen Y. Does vitamin D deficiency increase the severity of COVID-19? Clin Med 2020 Jun 5. DOI: 10.7861/clinmed.2020-0301.

69. Debuc B, Smadja DM. Is COVID-19 a New Hematologic Disease? Stem Cell Rev and Rep. 2020. https://doi.org/10.1007/s12015-020-09987-4.

70. Henry MB, Vikse J, Benoit S, Favaloro EJ, Lippi G. Hyperinflammation and
Derangement of Renin-AngiotensinAldosterone System in COVID-19: a novel hypothesis for clinically suspected hypercoagulopathy and microvascular immunothrombosis. Clin Chim Acta 2020 Apr; PII: S0009-8981(20)30183-2. DOI: https://doi.org/10.1016/j.cca.2020.04.027.

\section{Autor correspondente}

José Erivelton de Souza Maciel Ferreira. Rua Antônia Vicente, Baturité-CE 62.760-000, +5585998116578, eriveltonsmf@gmail.com.

Submissão: 2021-05-14

Aprovado: 2021-06-12 